DOE/ER/45930-4

\title{
QUANTUM MONTE CARLO STUDY OF ELECTRON CORRELATION IN HETEROSTRUCTURE QUANTUM DOTS
}

FINAL TECHNICAL REPORT

\author{
Mei-Yin Chou \\ School of Physics \\ Georgia Institute of Technology \\ Atlanta, GA 30332-0430
}

June 30, 2006

PREPARED FOR THE U.S. DEPARTMENT OF ENERGY

UNDER GRANT NUMBER DE-FG02-01ER45930 
The goal of this project is to study electron correlation in a confined geometry (quantum dots) within the two-dimensional quantum well in the sandwiches of two semiconductor materials. For these systems one is able to tune the electronic properties by controlling the size and the electron number, creating tremendous potential for novel applications. Much effort in this emerging field has been devoted to producing entangled states that are required for quantum information processing. At the same time, new physical phenomena have emerged from these artificial structures. Adding electrons to a quantum dot is more complicated than filling up discrete energy levels due to electron correlation. Therefore, our project is focusing on employing the state-of-the-art quantum Monte Carlo methods to study the electron-electron interaction. A close examination of the breakdown of Hund's rules and electron localization has been conducted in our simulations. The results are summarized below.

\section{INTRODUCTION}

Modern solid-state technology enables the fabrication of quasi-two-dimensional structures, called quantum dots, at semiconductor interfaces that confine a controllable number

of electrons [1, 2]. Tunnelling conductance [3] and capacitance experiments [4] suggest properties similar to natural atoms such as shell structures and ground states determined by Hund's Rules for quantum dots with strong confinement. In the regime of current experimental investigations, fermion liquid-like behavior dominates and it is appropriate to consider a picture of $\mathrm{N}$ particles moving around in a mean field with the single-particle orbitals being filled. Calculations based upon various approximations such as the Hartree-Fock theory $[5,6]$ and the density functional theory $[7-10]$ can provide reasonable results. Very accurate results for quantum dots are obtainable by using the configuration interaction (CI) approach [11-13]. However, this is limited to quantum dots containing only a few electrons and with moderate strength of confinement provided by either an electrostatic field or a strong magnetic field. As $\mathrm{N}$ increases or for quantum dots in which the electron-electron interaction dominates, the number of configurations to be included in a CI calculation increases so rapidly that the method becomes computationally too expensive. In this paper, we show that the quantum Monte Carlo (QMC) methods can yield results as accurate as the CI method while maintaining a much lower computational cost. Hence, the QMC methods 
enable us to investigate the electron correlation in quantum dots with a large number $\mathrm{N}$ of electrons and strong electron-electron interaction unprecedented by the CI calculations [14-17].

The electronic structure of a quantum dots strongly depends on the confinement strength. As the electron density in a quantum dot is lowered by weakening the confinement, the independent-particle picture will break down and the Hund's rules will be violated. It is interesting to know where this breaking-down occurs and what type of new ground states are then possible. A further reduction in electron density will lead to the spatial localization of electrons, forming a Wigner molecule. Its counterpart in two-dimensional infinite system is called Wigner crystal [18]. According to Monte Carlo simulations [19], such a transition occurs at the density corresponding to a Wigner-Seitz radius of $r_{s}=37 a_{B}^{*}\left(a_{B}^{*}\right.$ is the effective Bohr radius), preceded by a transition to the polarized state [20]. Chui and Tanatar [21] later showed that breaking the translational invariance from impurities significantly lowers the critical $r_{s}$ for this liquid $\leftrightarrow$ solid transition to $r_{s}=7.5 a_{B}^{*}$. Therefore, in a finite system like a quantum dot, one can expect a higher critical density (smaller $r_{s}$ ). A path-integral Monte Carlo simulation [15] suggested the onset of this Fermi liquid to Wigner molecule transition at $r_{s}=4 a_{B}^{*}$, regardless of the electron numbers. Spin-and-space unrestricted Hartree-Fock calculation [6] showed a weak Wigner molecule in $N=6$ quantum dot at $\hbar \omega=5 \mathrm{meV}$, corresponding to a even higher density. Reimann et al. [11] argued in a CI calculation that such a transition could not occur at $r_{s} \leq 4 a_{B}^{*}$ for $N=6$ and the critical density depends on electron numbers confined in a quantum dot.

Another interesting topic to be investigated is the spatial configuration of those localized electrons. In two-dimensional infinite systems it is a perfect triangular lattice [19], while in quantum dots it should be a compromise between the triangular lattice and the confinement shape. Some Monte Carlo simulations [22-24] suggested a shell structure in the spatial distribution of electrons in a parabolic quantum dot, with the central region being close to a triangular lattice. Saint Jean et al. [25] presented experimental results on macroscopic two-dimensional Wigner islands, which consist of electrostatically interacting charged balls with millimeter size. There are differences among those results though, since there are more meta-stable states with smaller energy gaps to the ground state as $\mathrm{N}$ increases.

In the following sections, we first briefly describe the QMC methods used in our calculation, the validity of which is established by comparing our results with those obtained 
by the CI method for a small number of electrons. The evolution of the ground state in quantum dots containing up to 13 electrons under a decreasing harmonic confinement is presented. Information on internal electronic structure are revealed by analyzing the spatial distribution of charge density and pair density.

\section{MODEL AND METHODS}

\section{A. The Hamiltonian}

The model Hamiltonian for $\mathrm{N}$ electrons confined in a potential well under zero magnetic field is

$$
H=\sum_{i=1}^{N}\left[-\frac{\hbar^{2}}{2 m^{*}} \nabla_{i}^{2}+V\left(\mathbf{r}_{\mathbf{i}}\right)\right]+\frac{e^{2}}{\epsilon^{*}} \sum_{i>j} \frac{1}{\left|\mathbf{r}_{\mathbf{i}}-\mathbf{r}_{\mathbf{j}}\right|},
$$

where $m^{*}$ is the effective electron mass, $\epsilon^{*}$ the effective dielectric constant. As usual, a harmonic confining potential is chosen for this work

$$
V\left(\mathbf{r}_{\mathbf{i}}\right)=\frac{m^{*}}{2} \omega^{2} r_{i}^{2}
$$

The noninteracting single particle energies are given in terms of the principal quantum number $n$ and angular momentum quantum number $l$ through $[26,27]$

$$
E_{n l}^{s p}=\hbar \omega(2 n+|l|+1)
$$

with $n=0,1,2, \cdots$, and $l=0, \pm 1, \pm 2, \cdots$. Hence the energy level $k \hbar \omega$ is $k$-fold degenerate.

We use effective atomic units hereafter unless specified otherwise. For the GaAs quantum dots considered here, we have $\epsilon^{*}=12.4$ and $m^{*}=0.067 m_{e}$. The length unit is then the effective Bohr radius $a_{B}^{*}=\frac{\varepsilon}{m^{*} / m_{e}} a_{B} \simeq 97.93 \AA$ and the energy unit is the effective Hartree $H^{*}=\frac{m^{*} / m_{e}}{\varepsilon^{2}} H \simeq 11.86 \mathrm{meV}$. One important dimensionless parameter is the ratio of the typical Coulomb interaction $e^{2} /\left(\epsilon l_{0}\right)$ to the ground-state energy $\hbar \omega$ of the harmonic oscillator, $\lambda \equiv e^{2} /\left(\epsilon l_{0} \hbar \omega\right)$, where $l_{0}=\sqrt{\hbar / m^{*} \omega}$ is the oscillator length. It is easy to show that

$$
\frac{\hbar \omega}{H^{*}}=\frac{1}{\lambda^{2}}
$$

In our calculation $\lambda$ was allowed to vary from 1.89 (corresponding to $\hbar \omega=3.32 \mathrm{meV}$ ) to 10 for most systems, while for some small $N$, we carried out our calculations up to $\lambda=20$. 


\section{B. Quantum Monte Carlo methods}

The variational Monte Carlo (VMC) method is based on the combination of the variational principle and the Monte Carlo evaluation of multi-dimensional integrals. An upper bound for the exact ground-state energy comes from the expectation value of the Hamiltonian with respect to a specific electronic state, $E[\Psi]=\int \Psi^{*} H \Psi d \mathbf{x} / \int \Psi^{*} \Psi d \mathbf{x}$, obtained by averaging the local energy $E_{L}\left(\mathbf{r}_{\mathbf{1}} \cdots \mathbf{r}_{\mathbf{N}}\right) \Psi \equiv H \Psi$ over many Monte Carlo steps.

Diffusion Monte Carlo (DMC) is more accurate in that it projects the ground state out of a trial wave function, while we can make the statistical error to be arbitrarily small by performing more Monte Carlo steps and the only systematic error comes from the approximate requirement of boundary conditions. Since there is no magnetic field, one can use the fixed-node approximation $[28,29]$ within which the ground state is supposed to have the same nodal surfaces as the trial wave function.

The accuracy of Monte Carlo calculations and the computational cost depend, to some extent, on the quality of the trial wave function. In our calculations, a trial wave function with several free parameters are used (see below). In the first step, these parameters are optimized to minimize the fluctuations of the local energy via the variational Monte Carlo method. In the second step, the optimized trial wave function is used as an input of the diffusion Monte Carlo calculation to further lower the ground-state energy. Both methods allows us to estimate the errors directly.

The trial wave functions for our Monte Carlo simulations are in the form of a sum of several Slater determinants multiplied by a generalized Jastrow function,

$$
\Psi_{L, S, S_{z}}\left(\mathbf{r}_{\mathbf{1}} s_{1 z}, \cdots, \mathbf{r}_{\mathbf{N}} s_{N z}\right)=\left(\sum_{m} \beta_{m} D_{m}\right) J\left(r_{i j}\right),
$$

where the Slater determinants are constructed from single particle orbitals $\psi_{n l \sigma}$ with principal quantum number $n$, angular momentum $l$ and spin projection $\sigma$, obtained from the density-functional calculations with the local spin density approximation. A Slater deter-

minant is a common eigenstate of operators $\hat{L}^{2}$ and $\hat{S}_{z}$. The parameters $\beta_{m}$ may depend on each other via the requirement that $\Psi_{L, S, S z}$ be a simultaneous eigenstate of the total spin operator $\hat{S}^{2}: S^{2} \Psi_{L, S, S_{z}}=S(S+1) \Psi_{L, S, S_{z}}$. Consider, for example, the ground state of ten electrons with $[\mathrm{L}, \mathrm{S}]=[0,0]$. The first six electrons occupy the single-particle orbitals 
TABLE I: Comparison of VMC and DMC energies with the energies obtained by CI method for $\mathrm{N}=4$. The energy is in units of the effective Hartree. The numbers in parentheses are statistical errors in the last digit.

\begin{tabular}{|c|c|c|c|c|c|c|}
\hline \multicolumn{4}{|c|}{$L=0, S=1$} & \multicolumn{3}{|c|}{$L=2, S=2$} \\
\hline$\lambda$ & VMC & DMC & $\mathrm{CI}$ & VMC & DMC & CI \\
\hline 2 & $3.4063(3)$ & $3.40421(2)$ & 3.40450 & $3.5636(1)$ & $3.56306(1)$ & 3.56338 \\
\hline 4 & $1.1923(2)$ & $1.18995(1)$ & 1.18952 & $1.2102(1)$ & $1.20977(1)$ & 1.20978 \\
\hline 6 & $0.65745(9)$ & $0.655744(4)$ & 0.655439 & $0.66164(6)$ & $0.661194(2)$ & 0.661181 \\
\hline 8 & $0.43381(7)$ & $0.432433(3)$ & 0.432338 & $0.43526(5)$ & $0.434682(2)$ & 0.434692 \\
\hline 10 & $0.31685(7)$ & $0.314178(2)$ & 0.314120 & $0.31573(3)$ & $0.315377(1)$ & 0.315323 \\
\hline 15 & $0.17795(3)$ & $0.176905(1)$ & 0.176961 & $0.17785(3)$ & $0.177338(1)$ & 0.177320 \\
\hline 20 & $0.11979(5)$ & $0.118307(1)$ & 0.118501 & $0.12019(5)$ & $0.118460(1)$ & 0.118503 \\
\hline
\end{tabular}

$\left(n, l, \sigma_{z}\right)=(0,0, \pm 1)$ and $(0, \pm 1, \pm 1)$ to form a compact core with vanishing spin and angular momentum. The other four electrons will occupy the orbitals $(1,0, \pm 1)$ or $(0, \pm 2, \pm 1)$. The following three combinations yield states with $\mathrm{L}=0$ and $S_{z}=0:(0,2, \pm 1)$ and $(0,-2, \pm 1)$; $(1,0, \pm 1),(0,2,-1)$, and $(0,-2,1) ;(1,0, \pm 1),(0,2,1)$, and $(0,-2,-1)$. The first configuration is an eigenstate of $\hat{S}^{2}$ with $S=0$. The second and the third configurations are to be combined together with $\beta_{2}=\beta_{3}$ in order to form an eigenstate of $\hat{S}^{2}$ with $S=0$.

The Jastrow function employed in our present calculations takes the following form

$$
J\left(r_{i}, r_{j}, r_{i j}\right)=\Pi_{i} \exp \left(A_{i}\right) \Pi_{i, j} \exp \left(B_{i j}\right) \Pi_{\alpha i j} \exp \left(C_{\alpha i j}\right)
$$

where $A_{i}$ are for electron-center correlation, $B_{i j}$ for electron-electron correlation, and $C_{\alpha i j}$ for (electron pair)-center correlation. The cusp conditions and the long-range behavior of the electron-electron interaction have been built into $A_{i}, B_{i j}$ and $C_{\alpha i j}$, the detailed expressions of which can be found in Ref. 17. We find that this Jastrow function is not yet flexible enough to yield a very accurate variational ground state, but it is sufficient for the guide function in a fixed-node DMC calculation after the parameters are optimized via the VMC calculation (see below). 


\section{Comparison with CI results}

In Table I, we presented the ground-state energies obtained by our VMC and DMC calculations for $\mathrm{N}=4$ together with those obtained by CI method from Ref. 13. The DMC energies are lower than the VMC energies because the DMC is an improvement over the VMC. Both differ in the third significant digits. This can serve as an estimate of the quality of our trial functions in describing the correlation effects. In the CI calculations, 24348 basis functions (Slater determinants) were used for the [1,0] state, while 8721 basis functions were used for the $[2,2]$ state. Our DMC energies agree in general with the CI energies in the first four significant digits. For $\lambda=20$ where the electron-electron correlation is strongest in our calculation, our DMC energies are even lower than the corresponding CI energies, indicating that more basis functions are needed in a CI calculation for the energy to converge up to the fourth significant digit. Our DMC results are also more accurate than those obtained by using the path-integral Monte Carlo method in Ref. 15.

\section{RESULTS AND DISCUSSIONS}

\section{A. Ground states and Hund's rules}

The similarity of a quantum dot and a natural atom lies in the grouping of energy levels into shells. Like natural atoms, electrons in a quantum dot obey the Hund's rules when filling the single-particle orbitals. Hund's first rule favors the alignment of electron spin to increase their pairwise spacings to lower electron-electron interaction energy. Hund's second rule suggests that electrons should take larger angular momentums so the system can spread further in space to lower the electrostatic energy between an outer electron and the core. Using these rules we obtained the groun-state configurations for $N=3-13$ as listed in Table II.

In Fig. 1, we present $\Delta E$, the energy difference between some low-lying states and Hund's ground state, as a function of the interaction strength $\lambda$ for $N=3$ and $N=5-13$. For sufficiently small $\lambda$, where $\Delta E>0$, Hund's ground state is the true ground state for all listed $N$. The electronic configurations listed in Table II provide the correct zerothorder approximation. As $\lambda$ increases, the energy gaps between shells diminishes so that the electron-electron interaction energy becomes comparable with or even more dominant than 
the energy gaps between shells, the true ground state is a linear combination of many different configurations. For $\mathrm{N}=3$, a transition to the spin-polarized ground state $\left[1, \frac{1}{2}\right] \rightarrow\left[0, \frac{3}{2}\right]$ is observed at $\lambda \simeq 4.0$, where $\Delta E$ becomes negative. This is consistent with the CI calculations by Mikhailov [12]. For $\mathrm{N}=4$, although the VMC data in Table I indicates a ground state transition $[0,1] \rightarrow[2,2]$ at $\lambda \simeq 10.0$, the more accurate DMC and CI data do not show any ground state transition in the ranges of $0 \leq \lambda \leq 20$.

For $\mathrm{N}=6$, Egger et al. [15] reported a transition $[0,0] \rightarrow[0,1]$ at $\lambda=8$. Such a transition is absent in our result shown in Fig. $1(\mathrm{~d})$. With $\lambda=8$, our DMC calculations yield a ground state energy $0.9426 H^{*}$, significantly lower than $0.9433 \pm 3 H^{*}$ obtained by Egger et al. [15]. CI calculations from Ref. 30 confirmed that no ground transition occurs in the ranges $0 \leq \lambda \leq 10.0$. For $N=8$, Egger et al. [15] reported a transition $[0,1] \rightarrow[0,2]$ at $\lambda \simeq 8.0$. Fig. $1(\mathrm{f})$ indicates that state $[0,1]$ continue to be the ground state. Our groundstate energy at $\lambda=8.0,1.6079 H^{*}$, is also lower than the value of $1.6106 \pm 6 H^{*}$ obtained by

TABLE II: Ground-state electronic configurations predicted by Hund's rules for $N=3-13$ quantum dots. L and $\mathrm{S}$ are respectively the total angular momentum and total spin in the ground state.

\begin{tabular}{lll}
\hline \hline $\mathrm{N}$ & occupied orbitals $\left(n, l, \sigma_{z}\right)$ & {$[\mathrm{L}, \mathrm{S}]$} \\
\hline $\mathbf{3}$ & $(0,0, \pm 1)(0,1,1)$ & {$\left[1, \frac{1}{2}\right]$} \\
$\mathbf{4}$ & $(0,0, \pm 1)(0, \pm 1,1)$ & {$[0,1]$} \\
$\mathbf{5}$ & $(0,0, \pm 1)(0, \pm 1,1)(0,1,-1)$ & {$\left[1, \frac{1}{2}\right]$} \\
$\mathbf{6}$ & $(0,0, \pm 1)(0, \pm 1, \pm 1)$ & {$[0,0]$} \\
$\mathbf{7}$ & $(0,0, \pm 1)(0, \pm 1, \pm 1)(0,2,1)$ & {$\left[2, \frac{1}{2}\right]$} \\
$\mathbf{8}$ & $(0,0, \pm 1)(0, \pm 1, \pm 1)(0, \pm 2,1)$ & {$[0,1]$} \\
$\mathbf{9}$ & $(0,0, \pm 1)(0, \pm 1, \pm 1)(0, \pm 2,1)(1,0,1)$ & {$\left[0, \frac{3}{2}\right]$} \\
$\mathbf{1 0}$ & $(0,0, \pm 1)(0, \pm 1, \pm 1)(0, \pm 2,1),(1,0,1)(0,2,-1)$ & {$[2,1]$} \\
$\mathbf{1 1}$ & $(0,0, \pm 1)(0, \pm 1, \pm 1)(0, \pm 2, \pm 1)(1,0,1)$ & {$\left[0, \frac{1}{2}\right]$} \\
$\mathbf{1 2}$ & $(0,0, \pm 1)(0, \pm 1, \pm 1)(0, \pm 2, \pm 1)(1,0, \pm 1)$ & {$[0,0]$} \\
$\mathbf{1 3}$ & $(0,0, \pm 1)(0, \pm 1, \pm 1)(0, \pm 2, \pm 1)(1,0, \pm 1)(0,3,1)$ & {$\left[3, \frac{1}{2}\right]$} \\
\hline \hline
\end{tabular}



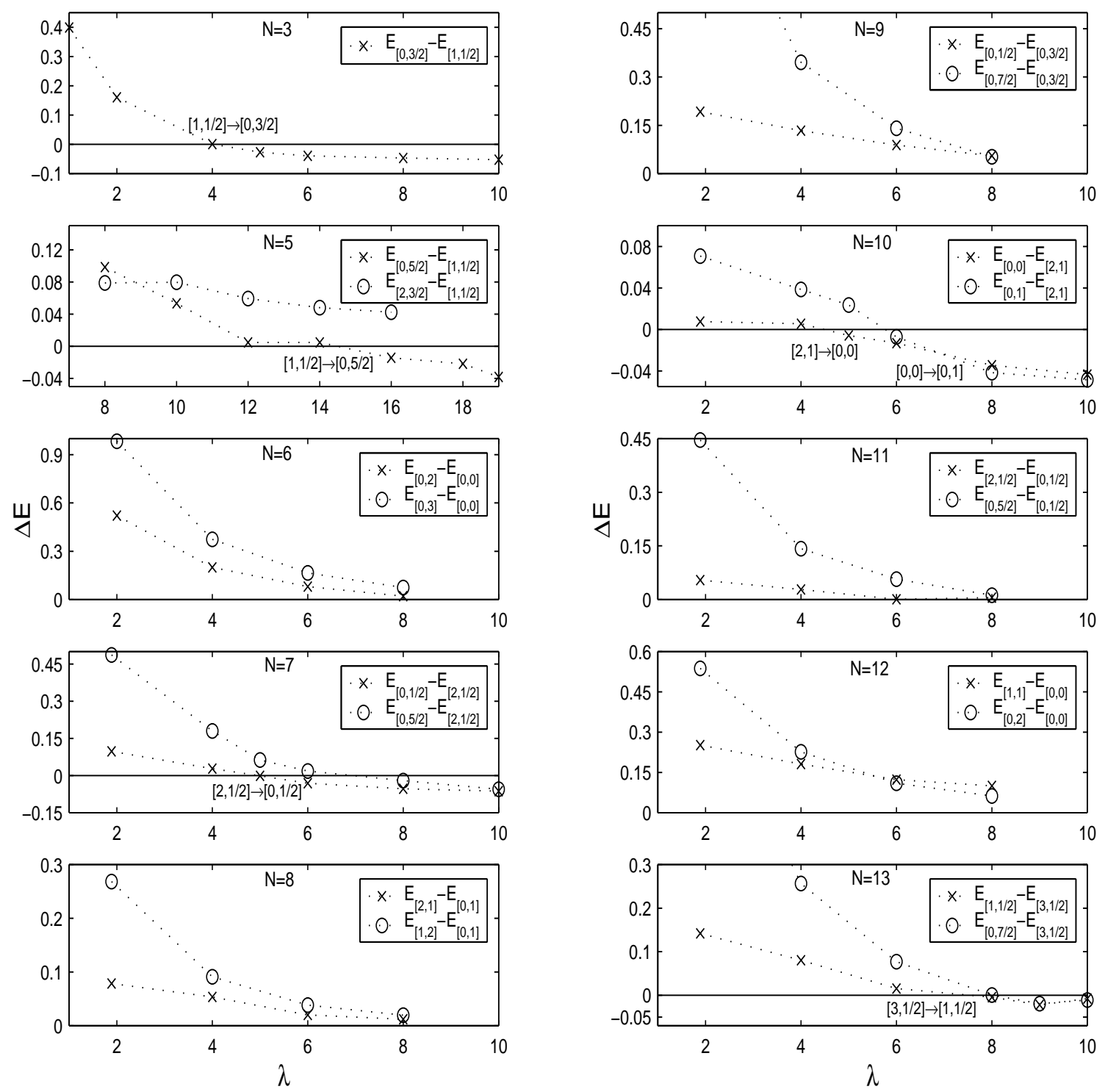

FIG. 1: The energy difference between some low-lying states and the Hund's ground state for N=3, $5-13$, in $\hbar \omega$.

Egger et al. [15]. Recall that the result from our VMC calculations for $\mathrm{N}=4$ quantum dot also favors the more spin-polarized state, but DMC results predict a ground state consistent with the Hund's rules.

An interesting observation from Fig. 1 is that the ground-state transition occurs only in systems with $N=3,5,7,10$ and 13, for which Hund's rules predict a non-vanishing $L$. When the transition takes place, the new ground state has either $L=0(N=3,5,7$, and 10) or $L=1(\mathrm{~N}=13)$. In the regime of $\lambda \sim 0, L$ comes from the independent motion of 


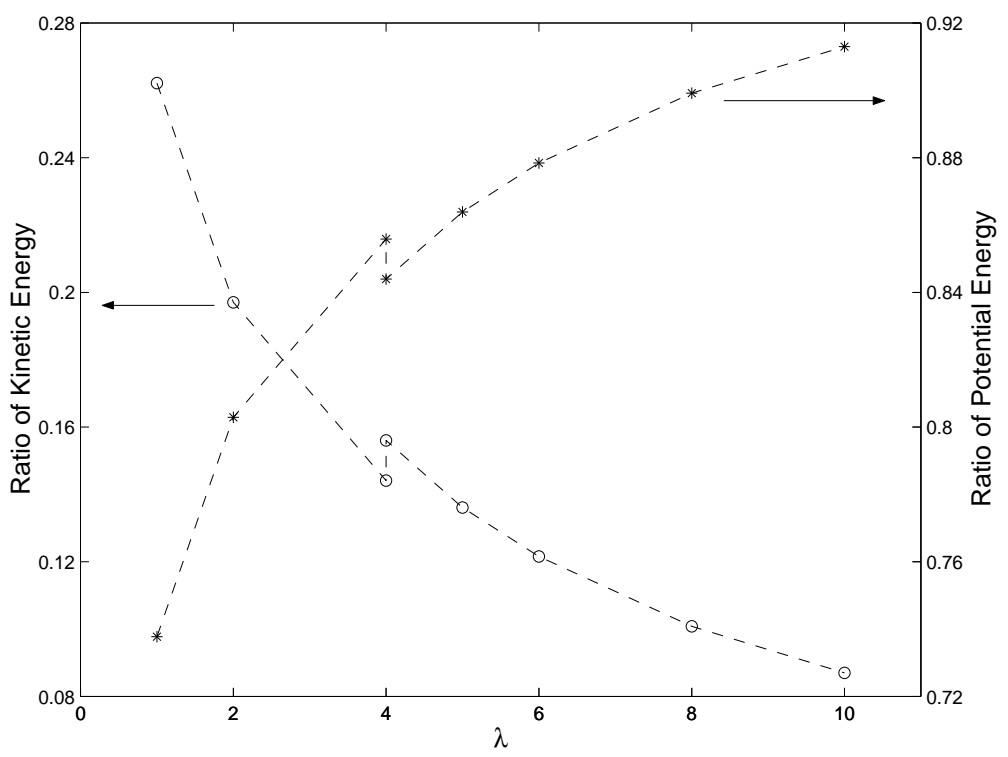

FIG. 2: The ratio of kinetic energy and potential energy to the ground state energy, respectively, as a function of $\lambda$ for $\mathrm{N}=3$.

the outer electron(s). For systems with strong correlation, Wigner molecules are formed, L describes the collective rotation of the molecule as a whole. In Fig. 2 the kinetic energy $E_{k}$ and the potential energy $E_{p}$ of the ground state of $\mathrm{N}=3$ are presented separately as a function of $\lambda$, which indicates a sudden drop in $E_{k}$ accompanied by an sudden increase of the same amount in $E_{p}$ when a ground state transition occurs. The total ground state energy varies continuously.

It is widely accepted that the appearance of a spin-polarized ground state be an evidence of a phase transition from a Fermi liquid to a Wigner molecule. Using the formula $r_{c}^{3}=$ $\lambda_{c}^{4} / \sqrt{N}$, we obtained the corresponding critical Wigner-Seitz radii $r_{c}=5.0 a_{B}^{*}$ for $\mathrm{N}=3$, $r_{c}>43.1 a_{B}^{*}$ for $\mathrm{N}=4$, and $r_{c}=25.8 a_{B}^{*}$ for $\mathrm{N}=5$. Hence our numerical calculations exclude the existence of a universal $r_{c}$ in few-electron quantum dots as claimed in Ref. 15.

We notice that the electronic states in a circular quantum dot differ from those in the bulk or in a noncircular quantum dot in one important aspect. In the bulk when electron-electron interaction is so strong that the wave functions exhibit isolated peaks, exchange of electrons is fully suppressed by the interaction. The eigenenergies and electron correlations become independent of electron spin (statistics). In a circular quantum dot, however, exchange of electrons is always possible via a rotation. Therefore eigenenergies and the electron correlations are always sensitive to spin. Consider, for example, two interacting electrons in 
a quantum dot with a weak confinement. Approximate analytical solutions (which become exact in the limit $\lambda \rightarrow \infty)$ gives a spin-unpolarized ground-state energy,

$$
E(S=0)=\frac{3}{2^{7 / 3}} \lambda^{-4 / 3}+\frac{\sqrt{3}}{4} \lambda^{-2}-\frac{1}{2^{11 / 3}} \lambda^{-8 / 3}-\frac{1}{2^{10 / 3} \sqrt{3}} \lambda^{-10 / 3}
$$

and a spin-polarized ground-state energy,

$$
E(S=1)=\frac{3}{2^{7 / 3}} \lambda^{-4 / 3}+\frac{\sqrt{3}}{4} \lambda^{-2}+\frac{3}{2^{11 / 3}} \lambda^{-8 / 3}+\frac{1}{2^{10 / 3} \sqrt{3}} \lambda^{-10 / 3},
$$

such that

$$
\xi=\frac{E(S=1)-E(S=0)}{E(S=0)} \sim \lambda^{-4 / 3},
$$

while in bulk or in noncircular quantum dots, $\xi$ decays exponentially with the increase of the interaction-strength parameter.

For $N=4$, we assert that the spin-polarized state $[0,2]$ cannot form the ground state of the Wigner molecule, for which the real-space equilibrium configuration is a regular square with the electrons at the vertices. In a regular square, a rotation of $\pi / 2$, which yields a phase factor $\exp (i \pi \mathrm{L} / 2)=1$ for $\mathrm{L}=0$, is equivalent to a cyclic permutation operating on the wave function of four fermions, which is an odd permutation and yields a phase factor of -1 . The only possibility is that the equilibrium configuration is a node of the wave function

and state $[0,2]$ is an excited state of the molecule. Using similar arguments, we can also show that the Wigner molecule with $\mathrm{N}=7$, the equilibrium geometric configuration of which is a centered hexagon, cannot have a spin-polarized ground state as $\lambda \rightarrow \infty$.

\section{B. Electronic structure at various confinement strengths}

The charge density is defined by

$$
\rho(r)=\left\langle\delta\left(\mathbf{r}_{\mathbf{i}}-\mathbf{r}\right)\right\rangle
$$

where $\langle\cdots\rangle$ denotes that the expectation value is taken with respect to the many-body wave function. $\rho(r)$ is independent of the angular coordinate for circular quantum dots and is useful in revealing the radial distribution. To further reveal the electronic structure of the system at different confinement strengths, we need to calculate the pair density

$$
P_{\sigma, \sigma^{\prime}}\left(\mathbf{r}, \mathbf{r}^{\prime}\right)=\frac{\left\langle\sum_{i \neq j} \delta\left(\mathbf{r}_{\mathbf{i}}-\mathbf{r}\right) \delta\left(\mathbf{r}_{\mathbf{j}}-\mathbf{r}^{\prime}\right) \delta_{\sigma, \sigma_{i}} \delta_{\sigma^{\prime}, \sigma_{j}}\right\rangle}{\left\langle\sum_{i} \delta\left(\mathbf{r}_{\mathbf{i}}-\mathbf{r}\right) \delta_{\sigma, \sigma_{i}}\right\rangle}
$$


with one electron fixed at a position $\mathbf{r}$. In this study we choose the position $\mathbf{r}$ at the location where the spin density with spin projection $\sigma$ has its maximum. The length scale is the effective Bohr radius $a_{B}^{*}$. The pair density is normalized such that $\int d \mathbf{r}^{\prime} P_{\sigma, \sigma^{\prime}}\left(\mathbf{r}, \mathbf{r}^{\prime}\right)=N_{\sigma^{\prime}}$ (=number of electrons with spin direction $\left.\sigma^{\prime}\right)$ if $\sigma^{\prime} \neq \sigma$ and $\int d \mathbf{r}^{\prime} P_{\sigma, \sigma}\left(\mathbf{r}, \mathbf{r}^{\prime}\right)=N_{\sigma}-1$.
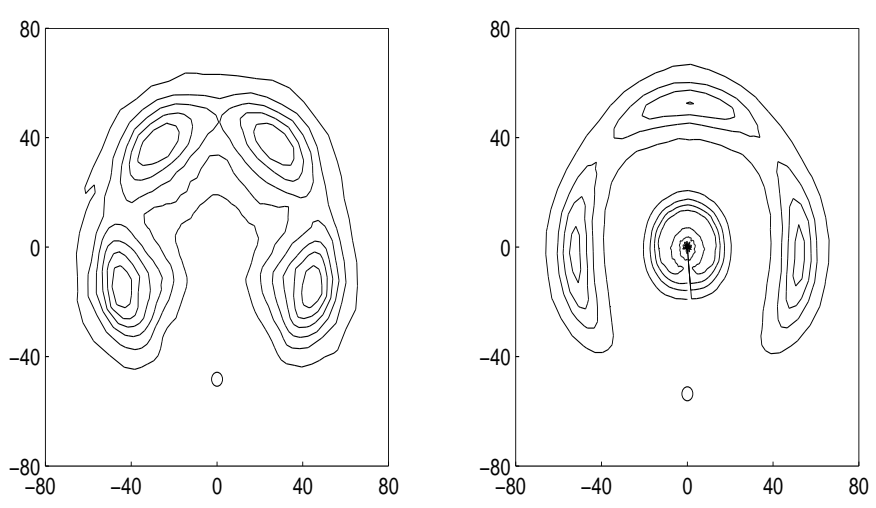

FIG. 3: Pair density distribution for $\mathrm{N}=5$ with $\lambda=16$ : (a) state $\left[0, \frac{5}{2}\right]$ and (b) state $\left[2, \frac{5}{2}\right]$. A spin-up electron is fixed at the place of the circle.

For $N \leq 5$ quantum dots, simple polygons are formed [22-24]. Our results for $N=3$ and 4 resemble those by Mikhailov [12] and strong localization can be observed. A clear pentagon can be seen in Fig. 3(a), featuring the pair density of the spin-polarized ground state $\left[0, \frac{5}{2}\right]$ at $\lambda=16$ in an $N=5$ quantum dot. The fixed electron is marked by a circle in the contour plot at the bottom of the graph. Also shown in Fig. 3(b) is the pair density of state $\left[2, \frac{5}{2}\right]$, which exhibits a centered square. This state has an eigenenergy $0.2550 H^{*}$ at $\lambda=16$, while the ground state energy is $0.2521 H^{*}$.

For $N \geq 6$ quantum dots, two- or more-shell structures should be expected in their Wigner molecules. We focus on $\mathrm{N}=7,10$ and 13, for which violations of Hund's rules are observed. Fig. 4 presents the charge density of their new ground states at (1) $\lambda=1.89$, appropriate for the experimental situation; (2) $\lambda=5$ for $N=7$ and 10 , and $\lambda=8$ for $N=13$, where the Hund's rule is violated and the transition takes place; and (3) $\lambda=10$, the largest $\lambda$ values in our calculations. The charge densities in Fig. 4 exhibit one peak when $\lambda$ is small. A shoulder emerges at a smaller $r$ when $\lambda$ is large enough for the ground-state transition to occur. The shoulder develops into a second peak as $\lambda$ is further increased, showing a stronger radial ordering.

In Fig. 5 , the pair density of state $\left[1, \frac{1}{2}\right]$ for $N=13$ simply shows a two-shell structure 

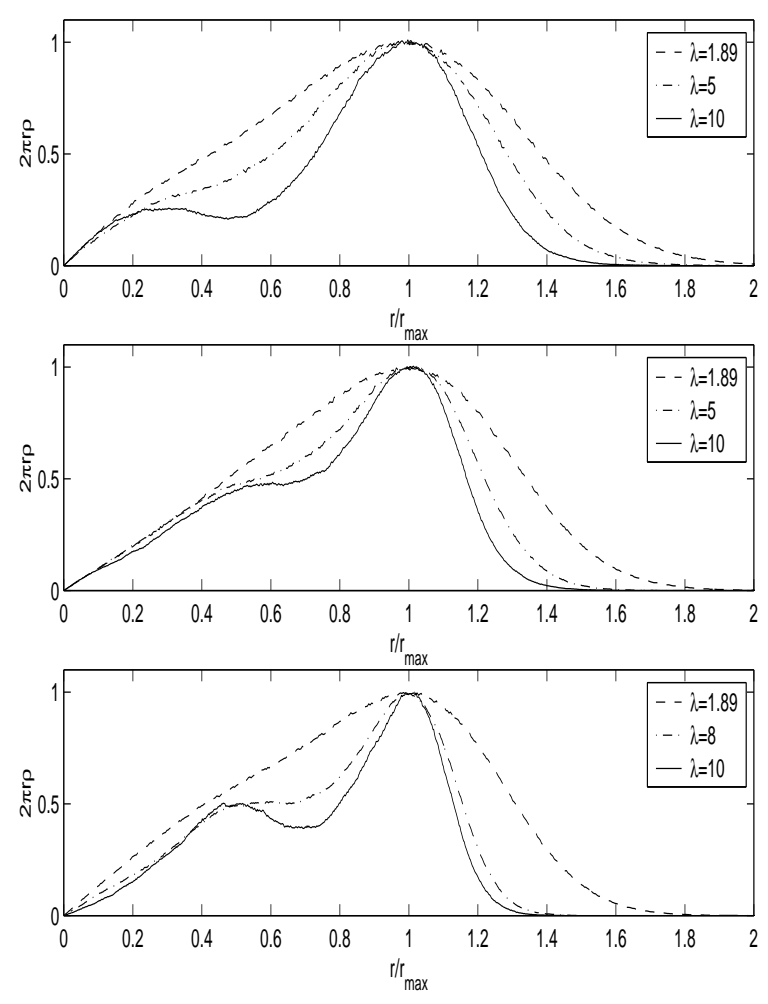

FIG. 4: Evolution of the charge density with increasing $\lambda$ : (a) state $\left[0, \frac{1}{2}\right]$ for $N=7$; (b) state $[0,0]$ for $N=10$; and (c) state $\left[1, \frac{1}{2}\right]$, for $N=13$. The radius $r$ is rescaled by $r_{m}$, the location of the maximum of $2 \pi r \rho(r)$.
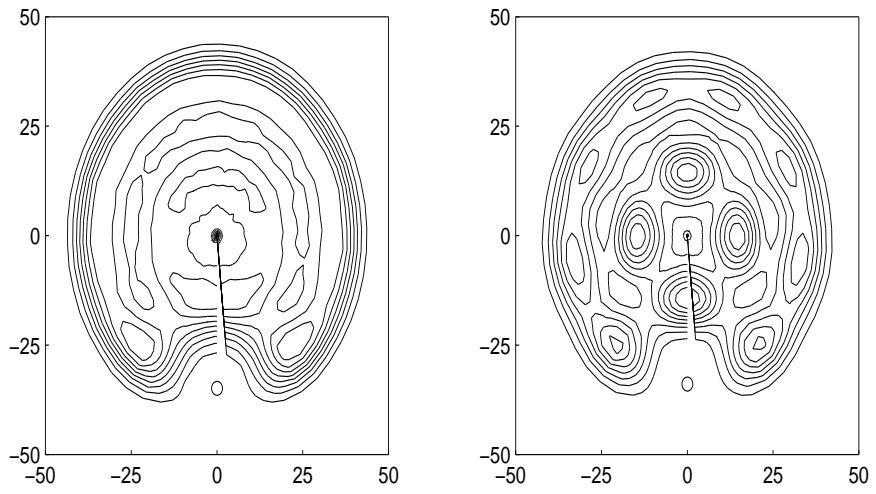

FIG. 5: Pair density distribution for $\mathrm{N}=13$ with $\lambda=8$ : (a) state $\left[1, \frac{1}{2}\right]$ and (b) state $\left[2, \frac{13}{2}\right]$.

without any pronounced peaks in each shell, while that of the spin-polarized state $\left[2, \frac{13}{2}\right]$ exhibits four peaks in the inner shell and nine peaks in the outer shell, in agreement with the structure determined by the classical-mechanics modeling in Ref. 23. Therefore, the spin-polarized states exhibit stronger intra-shell correlation than the unpolarized states. 

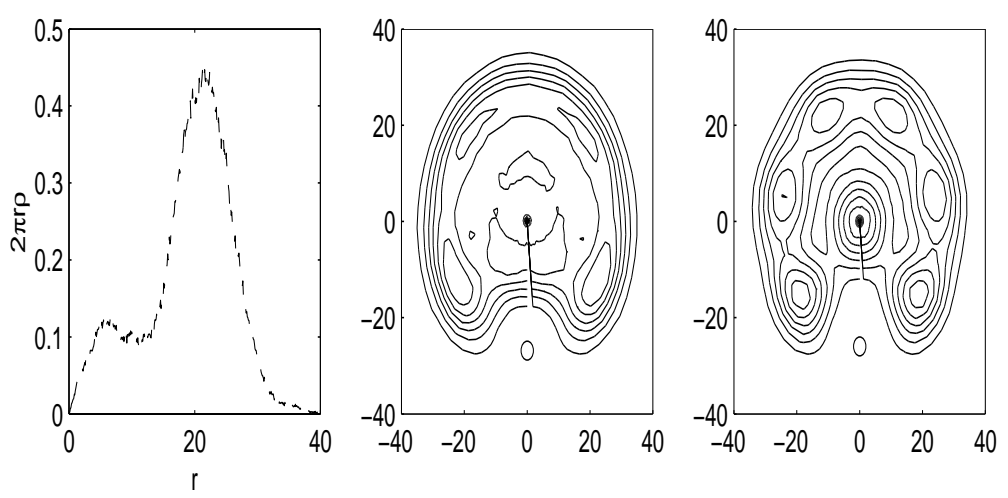

FIG. 6: For $\mathrm{N}=8$ with $\lambda=8$ : (a) radial distribution of the charge density for state $[0,1]$; (b) pair density distribution of state $[0,1]$; and (c) pair density distribution of state $[0,4]$.

Recall that two transitions are observed in Fig. 1 for $N=10$ and 13: the first transition is characterized by a drop in $L$, while the second one is characterized by an increase in $S$. This seems to support the idea of the two-step localization by Filinov [31], namely, radial ordering and orientational ordering, although Filinov's work suggests that the critical Wigner-Seitz radius for radial ordering to be $50 \sim 60 a_{B}^{*}$, much higher than the values of $6.2 a_{B}^{*}, 5.3 a_{B}^{*}$ and $9.6 a_{B}^{*}$ for $N=7,10$, and 13 quantum dots, respectively, in our results.

Even for $N=4,6,8,9,11$ and 12, which do not show any ground state transition in the $\lambda$ range of our investigation, the localization also occurs via the same two-step process. Fig. 6(a) presents the radial distribution of the charge density for the ground state $[0,1]$ of $\mathrm{N}=8$ with $\lambda=8$, which suggests a two-shell structure. Fig. 6(b) presents the corresponding pair density, which confirms the existence of two-shell structure, though its distribution inside a shell is still rather smooth, indicating a weak intra-shell correlation. Strong intrashell correlations can only be seen in the regime of a larger $\lambda$. Fig. 6(c) presents the pair density of the spin-polarized state $[0,4]$ with the same $\lambda$, which exhibits stronger intra-shell correlations due to the Pauli repulsion.

\section{CONCLUSIONS}

We have examined the ground-state energies and electron distributions in quantum dots containing up to 13 electrons. In the range of confinement strengths under investigation, violation of Hund's rules, characterized by a drop of $L$ in the ground state, is observed in 
quantum dots with the number of electrons $N=3,5,7,10$, and 13 . The transition can be understood as a tendency for a Wigner molecule to reduce its kinetic energy associated with the collective rotation. For $N=3$ and 5, a maximally spin-polarized ground state is found, but not for others. For $N=2,4,7$, etc., a completely spin-polarized ground state in the limit $\lambda \rightarrow \infty$ is prohibited by the particle exchange symmetry and the circular symmetry of the quantum dot. Hence a universal critical Wigner-Seize radius $r_{c}$ for the transition from a less correlated ground state to a localized ground state as the interaction strength increases does not exist in few-electron quantum dots with circular symmetry.

[1] M. A. Kastner, Rev. Mod. Phys. 64, 849 (1992), Phys. Today 46(1), 24 (1993).

[2] R. C. Ashoori, Nature (London) 379, 413 (1996), L. P. Kouwenhoven, T. H. Osterkamp, M. W. Danoesatro, M. Eto, D. G. Austin, T. Honda, and S. Tarucha, Science 278, 1788 (1997).

[3] U. Meirav, M. A. Kastner, and S. J. Wind, Phys. Rev. Lett. 65, 771 (1990).

[4] R. C. Ashoori, H. L. Stormer, J. S. Weiner, L. N. Pfeiffer, S. J. Pearton, K. W. Baldwin, and K. W. West, Phys. Rev. Lett. 68, 3088 (1992).

[5] H. M. Müller ans S. E. Koonin, Phys. Rev. B 54, 14532 (1996).

[6] C. Yannoules and U. Landman, Phys. Rev. Lett. 82, 5325 (1999).

[7] G. Vignale and M. Rasolt, Phys. Rev. Lett. 59, 2360(1987).

[8] M. Ferconi and G. Vignale, Phys. Rev. B. 50, 14722 (1994).

[9] M. Koskinen, M. Manninen, and S. M. Reimann, Phys. Rev. Lett. 79, 1389 (1997).

[10] K. Hirose and N. S. Wingreen, Phys. Rev. B 59, 4604 (1999).

[11] S. M. Reimann, M. Koskinen, and M. Manninen, Phys. Rev. B. 62, 8108 (2000).

[12] S. A. Mikkhailov, Phys. Rev. B. 65, 115312 (2002).

[13] S. A. Mikkhailov, Phys. Rev. B. 66, 153313 (2002).

[14] F. Bolton, Phys. Rev. B. 54, 4780(1996).

[15] R. Egger, W. Häusler, C. H. Mak, and H. Grabert, Phys. Rev. Lett. 82, 3320(1999).

[16] F. Pederiva, E. Lipparni, and C. J. Umrigar, Phys. Rev. B. 62, 8120(2000).

[17] C. Filipi and C. J. Umrigar, J. Chem. Phys. 105, 213-226 (1996).

[18] E. P. Wigner, Phys. Rev. 46, 1002(1934).

[19] B. Tanatar and D. M. Ceperly, Phys. Rev. B. 39, 5005 (1989). 
[20] F. Rapisarda and G. Senatore, Aust. J. Phys. 49, 161 (1996).

[21] S. T. Chui and B. Tanatar, Phys. Rev. Lett. 74, 458 (1995).

[22] F. Bolton and U. Rössler, Supperlatt. Microstruct. 13, 139 (1993).

[23] V. M. Bedanov and F. M. Peeters, Phys. Rev. B. 49, 2667 (1994)

[24] Y. Lai and I. Lin, Phys. Rev. E. 60, 4743 (1999).

[25] M. Saint Jean, C. Even, and C. Guthmann, Europhys. Lett. 55, 45 (2001).

[26] V. Fock, Z. Phys. 47, 446 (1928).

[27] C. G. Darwin, Proc. Cambridge Philos. Soc. 27, 86 (1930).

[28] C. J. Umrigar, M. P. Nightingale, and K. J. Runge, J. Chem. Phys. 99, 2865(1993).

[29] B. L. Hammond, W. A. Lester, and P. J. Reynolds, Monte Carlo Methods in ab initio Quantum Carlo Chemistry (World Scientific, Singapore, 1994)

[30] M. Cha and S. E. Yang, Phys. Rev. B. 67, 205312 (2003).

[31] A. V. Filinov et al., Phys. Rev. Lett. 86, 3851 (2001). 